\title{
GERMINOMA PINEAL COMO CAUSA DE PANHIPOPITUITARISMO: REPORTE DE UN CASO
}

\author{
Pineal Germinoma as a Cause of Panhypopituitarism: a case report
}

\author{
Dra. Elianny Aimeé Lantigua Güichardo*, Dra. Kathleen H. Ang**
}

Recibido: 6 Mayo, 2018 Aprobado: 15 Julio, 2018

Cómo citar: Lantigua Güichardo EA, H. Ang K. Germinoma pineal como causa de panhipopituitarismo: reporte de un caso. Ciencia Y Salud [Internet]. 14 sep.2018 [citado 14 sep.2018]; 2(3):41-6. Available from: https://revistas. intec.edu.do/index.php/cisa/article/view/1282

\section{Resumen}

Introducción: Los tumores de células germinales intracraneales constituyen un grupo infrecuente de neoplasias que afectan principalmente a la población pediátrica. Su presentación clínica varía en función de la localización y el tamaño del tumor. Las lesiones localizadas en la región pineal a menudo causan hidrocefalia obstructiva, mientras que las lesiones de localización supraselar, usualmente se asocian a disfunción del eje hipotálamo-hipofisario. Un tumor germinal de localización pineal que curse con endocrinopatías es una entidad extremadamente rara. Por tanto, reportamos el caso de un paciente masculino de 16 ańos de edad, con panhipopituitarismo secundario a un germinoma pineal.

Material y métodos: Este paciente fue referido al Departamento de Emergencias tras presentar hipernatremia en un análisis sanguíneo de rutina. Se presentó con polidipsia, poliuria y fatiga de un año de evolución. Las evaluaciones clínicas y pruebas de laboratorio reportaron

\footnotetext{
*Médico general

Cruz Roja Dominicana

eliannylantiguag@gmail.com

**Pediatra endocrinóloga

Escuela de Medicina de la Universidad de Yale, Departamento de Pediatría

kathleen.ang@yale.edu
}

diabetes insípida, panhipopituitarismo y hemianopsia bitemporal. El paciente recibió terapia de sustitución hormonal y tres meses después, la resonancia magnética de seguimiento reveló una masa en el área pineal que, a través de una posterior evaluación, fue diagnosticada como germinoma.

Conclusión: Luego de tratamiento con quimioterapia y radiación, hubo una resolución completa de la lesión. Sin embargo, no hubo mejoría clínica significativa y el paciente continúo necesitando terapia de reemplazo hormonal.

Palabras claves: Tumor de células germinales, germinoma pineal, panhipopituitarismo; diabetes insípida; terapia de reemplazo hormonal.

Abstract
Introduction: Intracranial germ cell tumors are rare neo-
plasms primarily affecting the pediatric population. The
clinical presentation varies by location and size. Lesions in
the pineal region often cause obstructive hydrocephalus,
while lesions in the suprasellar region typically present
with hypothalamic-pituitary axis dysfunction. A pineal
germ cell tumor presenting with endocrine disturbances
is a extremely rare entity. One case of a sixteen-year-old
male with panhypopituitarism secondary to a pineal ger-
minoma is reported.
Material and methods: This patient was referred to the
emergency department after his routine laboratory va-
lues showed hypernatremia. He presented with increased


thirst, polyuria, and fatigue of one-year duration. Clinical and laboratory evaluation revealed diabetes insipidus, panhypopituitarism, and bitemporal hemianopsia. He received hormone replacement therapy. Three months later, a follow-up magnetic resonance imaging revealed a mass at the pineal region diagnosed as germinoma.

Conclusion: After treatment with chemotherapy and radiation, he achieved complete resolution of the lesion. However, there was no marked clinical improvement and he continued to require hormone replacement therapy.

Keywords: intracranial germ cell tumor, pineal germinoma, panhypopituitarism, diabetes insipidus, hormone replacement therapy.

\section{Introducción}

Los tumores germinales intracraneales (TGIC) son neoplasias infrecuentes que solo representan alrededor de 3\% de los tumores cerebrales primarios de la población pediátrica. De acuerdo con sus características histológicas son clasificados como germinomas y tumores no germinomatosos. El subtipo más frecuente son los germinomas, los cuales representan dos tercios de los $\operatorname{casos}^{1}$.

Estos tumores afectan principalmente a niños y adolescentes, con una incidencia mayor entre los 10 y 12 años. Predominan en el sexo masculino con una relación de 2.5:1 ${ }^{1}$.

Por otro lado, se localizan en la línea media, principalmente en la región pineal y supraselar. Se encuentran en los ganglios basales, ventrículos, bulbo raquídeo o los hemisferios cerebrales con menor frecuencia ${ }^{1}$. Pueden aparecer como una lesión solitaria o múltiple, la presentación clínica es variable y depende de la localización del tumor y su tamaño ${ }^{2}$.

Las lesiones pineales tienden a causar síntomas como vómitos, cefalea, papiledema y diplopía debido a la obstrucción del acueducto del mesencéfalo, con un subsecuente aumento de la presión intracraneal. Los pacientes también pueden padecer somnolencia, ataxia, convulsiones y cambios conductuales ${ }^{1}$.

Los tumores en la región supraselar usualmente se asocian a disfunción del eje hipotálamo-hipofisario $^{3}$, y suelen ocasionar alteraciones endocrinológicas como diabetes insípida (DI), retraso puberal, hipotiroidismo y/o déficit de la hormona del crecimiento ${ }^{1}$. Los tumores bifocales aparecen en menor proporción (5-10\%) y cursan con endocrinopatías relacionadas a la localización supraselar ${ }^{2,3}$.

El diagnóstico de un TGIC se realiza basado en la combinación de las manifestaciones clínicas, la evidencia de hipopituitarismo, una evaluación de marcadores tumorales, una citología del líquido cefalorraquídeo, estudios de imágenes tales como tomografía axial computarizada (TAC); y una resonancia magnética (RMN) y/o biopsia del tumor. Sin embargo, las alteraciones endocrinológicas y neurológicas pueden aparecer antes de que exista evidencia radiológica del tumor ${ }^{1}$.

Es importante clasificar el tumor para la elección del tratamiento y determinar el pronóstico del paciente. Ya que los TGIC son sensibles a la radiación y quimioterapia, se suele utilizar una combinación de ambos como alternativa terapéutica ${ }^{1}$.

\section{Caso clínico}

Paciente masculino de 16 años de edad fue referido al Departamento de Emergencias tras presentar hipernatremia, en un análisis sanguíneo de rutina. Se presentó con polidipsia, poliuria y fatiga de un año de evolución. Tenía antecedentes de anorexia de 3 meses de evolución, la cual ocasionó una pérdida de peso de $2 \mathrm{kgs}$ y tuvo tres episodios de enuresis durante las 2 semanas anteriores a la visita. Sin embargo, negaba cefalea, cambios en la visión, convulsiones o síncope, náuseas o vómitos y no presentaba linfadenopatías. 
En la evaluación de su trayectoria en la curva de crecimiento, de los Centros para el Control y Prevención de Enfermedades (CDC), se observó un pobre crecimiento lineal ubicándose en el 6to percentil con $162 \mathrm{~cm}$ de altura y en el 12avo percentil para su peso, con $50.6 \mathrm{~kg}$. Su vello púbico se clasificó como Tanner grado IV, sus testículos como Tanner grado III, mientras que la longitud del pene era normal para su edad.

Pruebas de laboratorio revelaron una ligera anemia, hemoglobina en $12.9 \mathrm{~g} / \mathrm{dL}$ y niveles séricos de sodio y cloro elevados, con 164 y $128 \mathrm{mmol} / \mathrm{L}$, respectivamente. Además, se demostró insuficiencia parcial del eje hipotálamo-hipofisario con hiperprolactinemia:

\section{Cuadro 1: Resultados de pruebas de laboratorio}

\begin{tabular}{lcc}
\hline \multicolumn{1}{c}{ Prueba } & $\begin{array}{c}\text { Valores de labora- } \\
\text { torio }\end{array}$ & Valores de referencia \\
\hline FSH & $<0.1 \mathrm{mIU} / \mathrm{mL}$ & $0.85-8.74 \mathrm{mIU} / \mathrm{mL}$ \\
$\mathrm{LH}$ & $<0.1 \mathrm{mIU} / \mathrm{mL}$ & $0.29-4.77 \mathrm{mIU} / \mathrm{mL}$ \\
Testosterona & $79 \mathrm{ng} / \mathrm{dl}$ & $250-827 \mathrm{ng} / \mathrm{dL}$ \\
IGF-1 & $121 \mathrm{ng} / \mathrm{ml}$ & $229-522 \mathrm{ng} / \mathrm{mL}$ \\
TSH & $<0.0005 \mathrm{mUI} / \mathrm{L}$ & $0.3-4.2 \mathrm{mUI} / \mathrm{mL}$ \\
T4 Libre & $0.67 \mathrm{ng} / \mathrm{dL}$ & $0.8-1.4 \mathrm{ng} / \mathrm{dL}$ \\
Prolactina & $120 \mathrm{ng} / \mathrm{ml}$ & $0-14 \mathrm{ng} / \mathrm{mL}$ \\
\hline
\end{tabular}

La evaluación endocrinológica confirmó diabetes insípida, tras un incremento en la osmolalidad urinaria de $177 \mathrm{mosm} / \mathrm{kg}$ a $566 \mathrm{mosm} / \mathrm{kg}$, en respuesta a la administración intranasal de $10 \mathrm{mcg}$ de un análogo sintético de hormona antidiurética, dDAVP (1-Desamino-8d-Arginina Vasopresina).

Una prueba de estimulación con corticotropina (ACTH) mostró un incremento en el cortisol basal de $6.9 \mathrm{ug} / \mathrm{dl}$ a $17 \mathrm{ug} / \mathrm{dl}, 30$ minutos luego de la administración de $125 \mathrm{mcg}$ de un análogo sintético de ACTH. Además, el examen neuro-oftalmológico reveló hemianopsia bitemporal.

En la TAC craneal se evidenció un engrosamiento inespecífico del quiasma óptico, el cual también fue observado en la RMN cerebral. Se realizó una punción lumbar, y se tomaron muestras citológicas del líquido cefalorraquídeo tras la sospecha de malignidad. Se encontró un incremento en las células mononucleares y elevación del marcador tumoral gonadotropina coriónica humana ( $\beta$-hCG) de 15 $\mathrm{mIU} / \mathrm{L}$ (cuyo valor normal es $<5.0 \mathrm{mIU} / \mathrm{mL}$ ).

El paciente recibió tratamiento sustitutivo con hidrocortisona y desmopresina, seguido por tratamiento con levotiroxina y testosterona, el cual fue iniciado en las visitas de seguimiento posteriores a su alta médica.

Se realizó un RMN de control, a los seis meses que evidenció una lesión intracraneal localizada en la región pineal. La masa era homogénea, circunscrita, sólida y ovoide con $2.3 \mathrm{~cm}$ de diámetro. No hubo evidencia de hidrocefalia obstructiva. Se evaluó todo el eje cráneo-espinal con RMN y no se encontró metástasis tumoral. Una segunda punción lumbar mostró una elevación mayor de $\beta$-hCG (54 IU/L), lo cual, junto con los hallazgos imagenológicos, localización y características radiológicas del tumor, estableció el diagnóstico de germinoma.

Luego de cuatro ciclos de quimioterapia (carboplatino y etopósido) y bajas dosis de protonterapia hubo una resolución completa de la lesión. Sin embargo, el paciente no presentó una mejoría clínica significativa del panhipopituitarismo, por lo que continuó necesitando terapia de sustitución hormonal para su diabetes insípida, insuficiencia adrenal secundaria, hipotiroidismo secundario e hipogonadismo hipogonadotrópico.

En su visita más reciente, luego de dos años de la resolución de la lesión, el paciente se encuentra bien controlado con terapia de reemplazo hormonal y continuará su seguimiento con el Departamento de Endocrinología.

\section{Discusión}

Existen diferentes teorías sobre el origen de los TGIC. La teoría de Thelium plantea que surgen 
de una célula germinal primitiva totipotente que migra erróneamente durante el desarrollo embrionario. Las células germinales ectópicas evaden la apoptosis y posteriormente experimentan una transformación neoplásica, pero a pesar de tener el mismo origen muestran manifestaciones de acuerdo a su localización ${ }^{1,4,5}$.

Aunque las alteraciones endocrinológicas de nuestro paciente no están descritas como parte de la presentación clínica de los tumores pineales, al momento del diagnóstico resulta necesario considerar esta entidad, dada la presentación geográfica, apariencia radiológica y la elevación de marcador tumoral ${ }^{3,6}$.

La revisión de la literatura demuestra que los germinomas pineales son una entidad rara, y la mayoría de los casos reportados muestran síntomas de aumento de la presión intracraneal.

Avondet et al. ${ }^{7}$ reportan el caso de un paciente masculino de 20 años de edad con un germinoma pineal, que se presentó con diplopía y cefalea persistente y progresiva de un mes de duración. Ho et al. ${ }^{8}$ describen el caso de un paciente masculino de 16 años de edad con hidrocefalia obstructiva secundaria a un germinoma pineal.

Lin et al. ${ }^{9}$ estudiaron de manera retrospectiva doce niños diagnosticados con TGIC entre 1983 y 1995. Tres pacientes tenían lesiones pineales y todos cursaron con aumento de la presión intracraneal seguido de déficit visual. De la misma manera, Cormenzana Carpio et al. ${ }^{4}$ llevó a cabo un análisis retrospectivo de veinte pacientes diagnosticados con TGIC entre 1994 a 2014, de los cuales, 45\% tenían tumores pineales, y de estos, el $77.7 \%$ presentó cefalea y diplopía al momento del diagnóstico, y $44.4 \%$ presentaron alteraciones del campo visual.

Las manifestaciones de endocrinopatías en lesiones pineales son menos comunes ${ }^{6}$. Lin et al. ${ }^{9}$ describieron el caso de un paciente cuya única manifestación fue DI, tres años antes de que el tumor fuese radiológicamente detectable. Dodek et al. ${ }^{10}$ reportaron dos pacientes que cursaban con DI al momento del diagnóstico. Por otra parte, Tarng et al. ${ }^{11}$ describen el caso de un paciente que presentó DI, y tres años más tarde, desarrolló síntomas de aumento de la presión intracraneal.

A pesar de ser rápidamente fatales, los TGIC son tumores altamente curables con un buen pronóstico, si son tratados a tiempo. Aún existe controversia respecto a la modalidad de tratamiento óptimo; sin embargo, hay consenso en que la radiación craneoespinal debe ser considerada el gold estándar².

En algunos casos, la glándula pituitaria recupera su capacidad de secretar hormonas después del tratamiento del tumor. En otros pacientes, la disfunción endocrina persiste después del tratamiento y necesitan un reemplazo hormonal permanente. Esto puede deberse a los efectos directos ejercidos por el crecimiento tumoral, o debido a los efectos secundarios de la radioterapia y la cirugía; por tanto, es importante restringir el alcance de la cirugía y limitar la dosis de la radioterapia al mínimo, en la cual sea efectiva ${ }^{1}$. La quimioterapia y la radioterapia en nuestro paciente dieron como resultado la resolución completa de la lesión pineal. Sin embargo, los síntomas endocrinológicos de DI y la insuficiencia hipofisaria anterior, han persistido.

Aunque los TGIC son poco frecuentes, es importante que los médicos, especialmente los pediatras, estén familiarizados con esta enfermedad, es potencialmente curable. Es importante tener en cuenta el diagnóstico de TGIC cuando los niños, adolescentes y adultos jóvenes presenten alteraciones endocrinológicas y/o neurológicas, ya que pueden aparecer antes de que la detección radiológica del tumor sea posible. La mayoría de los TGIC responden a la radioterapia y la quimioterapia, y es necesario iniciar el tratamiento con rapidez para minimizar la morbilidad y la mortalidad ${ }^{1}$. 


\section{Bibliografía}

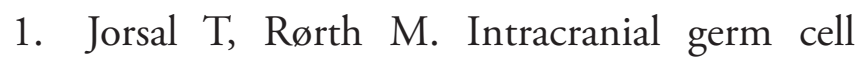
tumours. A review with special reference to endocrine manifestations. Acta Oncológica. 2012;51(1):3-9.

2. Krueger E, Invergo D, Lin J. Germinoma with Diffuse Subependymal Spread: A Case Report. Cureus. [Internet] 2016 [Citado el 2018 Jun 23]. Disponible en: https://www.researchgate. net/publication/304006173_Germinoma_ with_Diffuse_Subependymal_Spread_A_ Case_Report

3. Reisch N, Kühne-Eversmann L, Franke D, Beuschlein F, Mueller-Lisse U, Reincke M, et al. Intracranial Germinoma as a Very Rare Cause of Panhypopituitarism in a 23-year Old Man. Experimental and Clinical Endocrinology \& Diabetes [Internet] 2009 [Citado el 2018 Jun 23]117(7):320-3 Disponible en: https://www. researchgate.net/publication/23567969_ Intracranial_Germinoma_as_a_Very_Rare_ Cause_of_Panhypopituitarism_in_a_23year_Old_Man

4. Cormenzana Carpio M, Alvarez D, Hernandez Marquez C, Perez Martinez A, Lassaletta Atienza A, Lopez L, Intracranial germ cell tumours: A 21-year review. Anales de Pediatría (English Edition). 2017;86(1):20-27.

5. Lester L, Saran F, Hargrave D, Bodi I, Bassi S, Hortobagyi T. Germinoma with synchronous lesions in the pineal and suprasellar regions. Chld's Nervous System. [Internet] 2007 [citado el 2018 Jun 23];22(12):1513-1518. Disponible en: https://www.researchgate.net/ publication/6741797_Germinoma_with_synchronous_lesions_in_the_pineal_and_suprasellar_regions

6. Echevarria M, Fangusaro J, Goldman S. Pediatric Central Nervous System Germ Cell
Tumors: A Review. The Oncologist. [Internet] 2008 [citado el 2018 Jun 23];13(6):690-699. Disponible en: http://theoncologist.alphamedpress.org/content/13/6/690.full.pdf+html

7. Avondet F, Cabo F, Negrotto M, Centurion D. Tumor mixto de células germinales de la glándula pineal. Argentinian Journal of Radiology. [Internet] 2018 [Citado el 2018 Jun 23];82:144-147. Disponible en: https:// www.thieme-connect.com/products/ejournals/pdf/10.1055/s-0038-1639495.pdf

8. Ho C, Deruytter M. Dual Occurrence of Pineal Germinoma and Testicular Seminoma. Pediatric Neurosurgery. [Internet] 2006 [citado 2018 Jun 23].;42:38-44. Disponible en: https://www.karger.com/Article/Abstract/89508

9. Lin IJ, Shu SG, Chu HY, Chi CS. Primary Intracranial Germ-Cell Tumor in Children. Journal of the Chinese Medical Association. [Internet] 1997 [Citado el 2018 Jun 23];60:259-264. Disponible en: http://homepage.vghtpe.gov.tw/ -jcma/6005/600505.htm

10. Dodek A, Sadeghi-Nejad AB. Pineal Germinoma Presenting as Central Diabetes Insipidus. Clinical Pediatrics. [Internet] 1998 [citado el 2018 Jun 23];37(11):693-695. Disponible en: http://journals.sagepub.com/ doi/abs/10.1177/000992289803701109?journalCode=cpja

11. Tarng D, Huang T. Diabetes Insipidus as an Early Sign of Pineal Tumor. American Journal of Nephrology [Internet] 1995 [citado el 2018 Jun 23];15:161-164. Disponible en: https:// www.karger.com/Article/Abstract/168823\#

12. Kakani B, Karmarkar S, Deopujari E, Shah Rajan M, Bharucha E, Muzumdar G, Germinoma of fourth ventricle: A case report and review of literature. Journal o Pediatric Neurosciencie. 2006;1(1):33-35. 
13. Ballesteros MD, Duran A, Arrazola J, Redondo MJ, Bordiu E, De Abajo S, et al. Primary intrasellar germinoma with synchronous pineal tumor. Neuroradiology. 1997;39(12):860-862.

14. Pomarede R, Czernichow P, Finidori J, Pfister A, Roger M, Kalifa C, et al. Endocrine aspects and tumoral markers in intracranial germinoma: An attempt to delineate the diagnostic procedure in 14 patients. The journal of pediatrics. 1982;101(3):374-378.
15. Diez, B. Tumores germinales intracraneales. Arch. neurol. neuroc. neuropsiquiatr. 2007; 13(1):16-24.

16. Pérez-García J. Germinoma intracraneal, 2 casos en varones adolescentes. Revista Española de Patología. [Internet] 2007 [Citado el 2018 Jun 23]; 40(4). Disponible en: http://www.patologia.es/volumen40/vol40-num4/40-4n06. htm 